\title{
Visualization of White Matter Tracts with Wrapped Streamlines
}

\author{
Frank Enders* \\ Neurocenter, \\ Dept. of Neurosurgery \\ Computer Graphics Group
}

\author{
Natascha Sauber ${ }^{\dagger}$ \\ Computer Graphics Group
}

\author{
Christopher Nimsky ${ }^{\S}$ \\ Dept. of Neurosurgery
}

\author{
Dorit Merhof* \\ Neurocenter, \\ Dept. of Neurosurgery \\ Computer Graphics Group
}

\author{
Marc Stamminger* \\ Computer Graphics Group
}

\author{
Peter Hastreiter \\ Neurocenter, \\ Dept. of Neurosurgery \\ Computer Graphics Group
}

University of Erlangen-Nuremberg, Germany
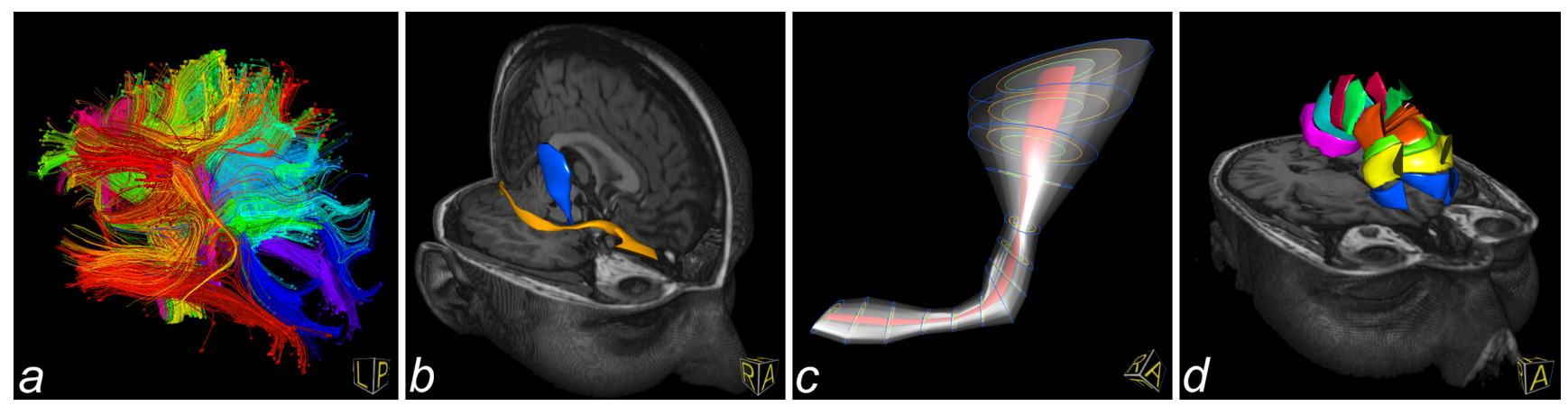

Figure 1: Samples generated with our diffusion tensor visualization application. (a) shows automatically clustered whole brain tracking with assigned colors. $(b)$ displays the optic tract (orange) and the pyramidal tract (blue) in combination with direct volume rendering of high resolution magnetic resonance data. $(c)$ is an example for safety estimation with the use of staggered hulls. $(d)$ shows color encoded cluster segments of the corpus callosum combined with $\mathrm{MRI}_{T 1}$ data.

\section{AbstraCt}

Diffusion tensor imaging is a magnetic resonance imaging method which has gained increasing importance in neuroscience and especially in neurosurgery. It acquires diffusion properties represented by a symmetric 2 nd order tensor for each voxel in the gathered dataset. From the medical point of view, the data is of special interest due to different diffusion characteristics of varying brain tissue allowing conclusions about the underlying structures such as white matter tracts. An obvious way to visualize this data is to focus on the anisotropic areas using the major eigenvector for tractography and rendering lines for visualization of the simulation results. Our approach extends this technique to avoid line representation since lines lead to very complex illustrations and furthermore are mistakable. Instead, we generate surfaces wrapping bundles of lines. Thereby, a more intuitive representation of different tracts is achieved.

CR Categories: I.3.8 [Computer Graphics]: Applications J.3 [Life and Medical Sciences]: Medical Information Systems

Keywords: Diffusion Tensor Imaging, Tractography, White Matter Tracts, Clustering

\footnotetext{
*e-mail: \{enders,merhof,stamminger\}@ informatik.uni-erlangen.de

†e-mail: nasauber@stud.informatik.uni-erlangen.de

¥e-mail: hastreiter@neurozentrum.imed.uni-erlangen.de

§e-mail: nimsky@nch.imed.uni-erlangen.de
}

\section{INTRODUCTION}

With the growing availability of diffusion tensor imaging (DTI) data [3], the need for comprehensive visualization is increasing. Especially, neurosciences are interested in meaningful 2D and 3D representations of this data since it allows additional conclusions about brain tissue supporting standard magnetic resonance imaging (MRI) sequences. DTI delivers information about nerve tracts (white matter tracts), such as the optic and pyramidal tract, which serve as connections between different areas of the brain and the spinal cord. In fact, a predominant part of the brain consists of such white matter. The parallel orientation of the underlying neuronal fibers leads to anisotropic water diffusion characteristics measured by DTI.

Fiber tracking approaches have become a widely accepted visualization technique for DTI evaluation indicating the location of white matter tracts within the human brain. In neurosurgery, fiber tracking results help to make diagnosis and therapy planning safer [25]. During surgery the integration of the tract information into the neuronavigation system allows more precise identification of critical nerve structures. Thereby, a visualization of the data is blended over the real situation seen through the microscope. In order to avoid disturbance of a clear view onto the operating field it is necessary to simplify the structure of the displayed objects.

Up to now, research has focused on reconstructing fiber tracts based on different approaches such as streamline tracking [2], tensor deflection [21] and tensor lines [30]. Another class of algorithms incorporates probabilistic information [4] or tries to reconstruct a continuous representation of the data [27].

From a surgical point of view the representation of white matter tracts is still insufficient and single streamlines are of minor inter- 
est. However, the whole fiber bundle taking into account the hull of the tract is highly relevant for planning since it shows the transition to functionally important structures which have to be prevented from injury in any case. A hull encompassing all fibers of a certain tract would at the same time show the course and the extent of the corresponding nerve structure such as the pyramidal tract.

In order to improve application in neurosurgery we present a new technique to support the identification of nerve tract borders. Based on tractography results, our approach generates surfaces corresponding to the underlying nerve tracts. This is achieved by determination of a subset of fibers out of the complete tracking which is related to a certain nerve tract. Subsequently, the proposed algorithm wraps the specified fibers with a surface that represents the transition between nerve tract and surrounding tissue.

The paper is organized as follows: In Section 2 related work is presented. Section 3 gives an introduction to the DTI data. Section 4 comprises the preprocessing steps tracking and bundling. Section 5 describes the presented algorithm. Visualization techniques are presented in Section 6 and discussed in Section 7.

\section{RELATED WORK}

Several approaches have been developed to access the information contained in DTI data. The easiest way is the visualization using 2D slices $[12,26]$. Thereby, the principle eigenvector related to the largest eigenvalue of each tensor is directly mapped to RGB color space. This is a very popular strategy since the images are directly related to well known slice images from MRI and computed tomography (CT).

Slice images with additionally encoded direction information are still hard to interpret. Therefore, 3D approaches have been developed widening the abilities to explore the data but simultaneously complicating interpretation due to arbitrary viewing direction occlusion and other difficulties. Furthermore, the higher dimension of tensor data is challenging when compared to scalar volume data. This has encouraged a variety of different strategies such as the visualization of each single tensor by rendering glyphs in various shapes $[32,17,13]$. Another approach is volume rendering for tensor data $[18,19]$. Thereby, tensor information is encoded to textures, which allows mapping of color and opacity for shading. Both strategies attempt to convey as much information as possible by utilizing the diverse features of the techniques like shape, size, color and transparency. This can easily lead to overloaded visualization.

Tractography, the probably best known strategy, reduces the data to the principal eigenvector, similar to color mapping in 2D. By incorporating streamline techniques known from flow visualization [6], an intuitive representation of DTI data is provided since the structures of interest are fiber tracts and the resulting lines are good analogons. This is reflected in many works which are focused on this strategy $[2,22]$. To improve visual perception, lines were extended to streamtubes [34] which can provide a better overall impression. Hyperstreamlines [8, 33] finally add mutable spatial structure to streamlines which increase the information content of a single line.

In order to assist the user to further explore DTI data, approaches have been proposed to cluster lines $[5,10]$. Due to the complexity of the underlying structure, it is hard to identify single tract systems without visual support. Clustering strategies use analytical methods to group sets of lines. This provides the necessary visual support using color encoding to emphasize their context.

Finally, those clusters can be surrounded by a hull showing the border of the nerve tract as presented by Ding et al. [9]. Aiming at the generation of hulls, this approach starts from a seed point region, further on determines corresponding fiber segments and finally generates a convex hull. Contrary to that, we do not require a 2D seed point plane but works with arbitrary bundles.

\section{IMAGE DATA}

The idea of DTI is the acquisition of the diffusion property of water molecules instead of their quantity and binding, known from common MR. Generally, water molecules are moving which is known as Brownian Motion [11]. This movement is affected by cell shape and other factors like permeability of the cell membrane and accordingly, the diffusion property is affected as well. This is of special interest since the diffusion property allows conclusions about the underlying tissue structure in vivo. In particular, white matter shows a strong anisotropic diffusion characteristic which originates from the long, cylindric cell structure of white matter. Thereby, it can be differentiated from other brain tissues. Consequently, the direction of the measured diffusion allows estimating the orientation of neuronal fibers. High anisotropy and the corresponding major eigenvector thereby indicate the location and course of neuronal fibers. In contrast to that, isotropic diffusion occurs in tissue with round cell shapes or in fluid filled cavities like the ventricles. Areas of planar diffusion in-between can be found at fiber crossings or branchings.

Diffusion properties are represented by a symmetric second order tensor

$$
D=\left(\begin{array}{ccc}
D_{x x} & D_{x y} & D_{x z} \\
D_{x y} & D_{y y} & D_{y z} \\
D_{x z} & D_{y z} & D_{z z}
\end{array}\right)
$$

which requires to measure at least 7 complete MRI volumes. They are the basis for the computation of diffusion tensors. For each gradient direction a diffusion weighted image is acquired. In order to be able to specify a symmetric second order tensor, representing the diffusion property at a voxel, 6 of the 7 datasets represent defined gradient directions and one serves as an anatomical reference without directional information [3].

All the datasets used in this work were measured using a Siemens MR Magnetom Sonata Maestro Class 1.5 Tesla scanner. The specifications of the gradient system were a field strength of up to $40 \mathrm{mT} / \mathrm{m}$ (effective $69 \mathrm{mT} / \mathrm{m}$ ) and a slew rate of up to $200 \mathrm{~T} / \mathrm{m} / \mathrm{s}$ (effective $346 \mathrm{~T} / \mathrm{m} / \mathrm{s}$ ) for clinical application. We chose a field of view of $240 \mathrm{~mm}$ resulting in a voxel size of $1.875 \times 1.875 \times 1.9 \mathrm{~mm}^{3}$. For each of the six gradient directions $( \pm 1,1,0),( \pm 1,0,1),(0,1, \pm 1)$ and the reference image sixty slices with no intersection gap and an acquisition matrix of $128 \times 128$ were measured. Additionally, a $\mathrm{MRI}_{T 1}$ sequence $(256 \times 256 \times 160$ voxels $)$ was consecutively obtained for each patient with unchanged position of the head. This avoided registration of the datasets for a combined examination which would have been necessary in case of misalignment.

\section{Preprocessing}

\subsection{Streamline Tracking}

Streamline tracking algorithms in general have several steps in common encompassing seed point selection, fiber propagation and termination strategies [24]. Starting from certain seed points or regions of interest (ROIs), streamline techniques are applied to propagate the fiber until a stopping criterion is reached. This can either be a threshold based on fractional anisotropy (FA) [2, 23] or curvature [2]. In the first case, fiber propagation is stopped if the direction of fastest diffusion is not well defined. The latter criterion follows the assumption that fibers are somewhat stiff which prevents the tracking algorithm from following unlikely paths.

For the definition of seed regions, different approaches exist: Seeds may be automatically located in regions with high anisotropy. If the user additionally specifies ROIs, only those fibers are displayed which run through the ROIs. As an advantage, fibers within the whole brain are precomputed and can be subject to different dynamic queries [1]. Another approach directly positions seed points 
within the ROIs which is assumed to consume less computation time. However, if more tract systems within the same subject have to be investigated, the first approach may be more appropriate. Additionally, if the ROI is located in areas of higher uncertainty of diffusion direction, the tracking result may be more reliable if tracking is started in regions of high directional diffusion.

Fiber propagation is accomplished using streamline approaches known from flow computations. In most cases, trilinear tensor interpolation, computation of the eigensystem and numerical integration are used in each propagation step. To derive a tensor at an arbitrary position within the dataset, component-wise trilinear interpolation of the tensor entries is commonly used providing better results than interpolation of the principal eigenvectors [19]. The computation of the eigensystem of the interpolated tensor provides the major eigenvector which correlates with the direction of fastest diffusion. Euler integration propagates the streamline by advancing in this direction, higher order integration schemes require repeated tensor interpolation and eigensystem computation to determine the direction of streamline propagation. We used the fourth-order Runge-Kutta method providing sufficient accuracy for tracking. Figure 2 gives a schematic outline of streamline propagation.

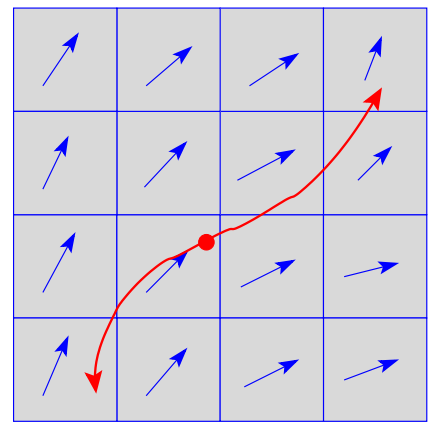

Figure 2: Schematic outline of the fiber tracking process. From a seed point trajectories are propagated according to the underlying diffusion direction until a termination criterion is reached.

\subsection{Bundling of streamlines}

Most applications based on DTI fiber tracts aim at investigating separate tract systems. In the human brain, a number of tract systems related to specific tasks can be distinguished, e.g. the corpus callosum which connects both hemispheres, the optic radiation connecting the optic tract and the primary visual cortex in the occipital lobe, or the pyramidal tract running from the motor cortex down to the spine. For extracting these tract systems two general approaches exist (see Figure 3):

- ROIs allow separating tract systems directly during or after tracking. According to anatomical knowledge or based on FA maps, the ROIs are defined and used as input for fiber tracking which returns only those fibers running through the ROIs.

- For automatic clustering of fibers from the whole brain, several characteristics are computed for each fiber such as the center of gravity, variance, covariance and curvature. Afterwards, fibers are clustered based on their attributes. The respective fibers of the received clusters build the separated bundles.

For our application we applied a strategy proposed by Brun et al. [5] which uses the NCut [29] algorithm for clustering. However, other clustering strategies might be applied as well.
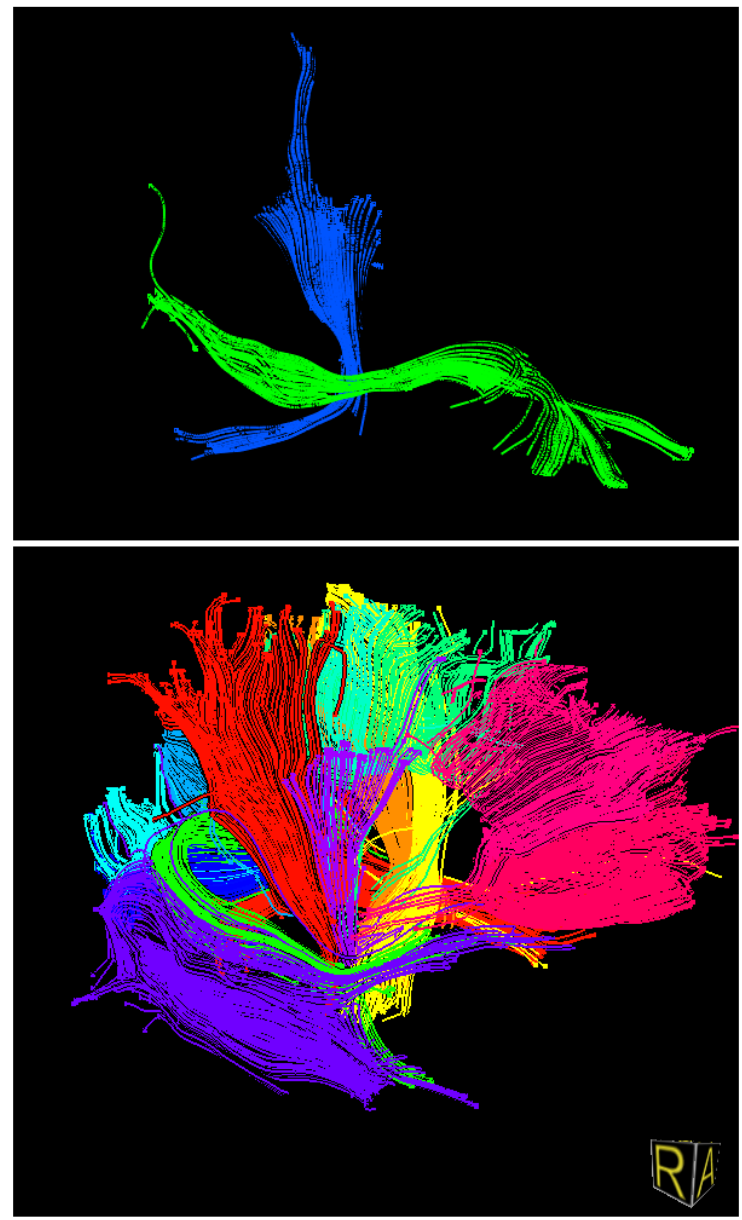

Figure 3: Examples of a fiber bundle using ROls (top) and clustering using an adapted version of the approach of Brun et al. [5] (bottom) in a healthy volunteer. In case of ROI bundling, the right pyramidal tract (blue) and the right optic tract (green) were selected. In the lower image only selected clusters related to prominent nerve tracts are shown. Minor clusters are masked for convenience. Both images are taken from the same point of view.

\section{WRAPPING OF FIBER BUNDLES}

In this section we describe the approach of wrapping streamlines. First, a center line is determined, averaging the fibers of the bundle. Thereafter, planes are specified which serve as a basis for boundary curves. Finally, the constructed curves are stitched together to obtain the final mesh forming a hull.

\subsection{Center Line}

The fibers received from the tracking algorithm consist of equidistant points and are terminated by a starting and an end point. Accordingly, the length of a fiber equals the number of points minus 1 times the distance between two points.

The center line of a fiber bundle averages the characteristics of the individual streamlines. It approximates the position and curvature of the complete cluster. Each single point of the centerline is computed by averaging the corresponding points of all fibers (i.e. the starting point of the centerline is the average of all starting points of the fibers, the second point of the centerline is the aver- 

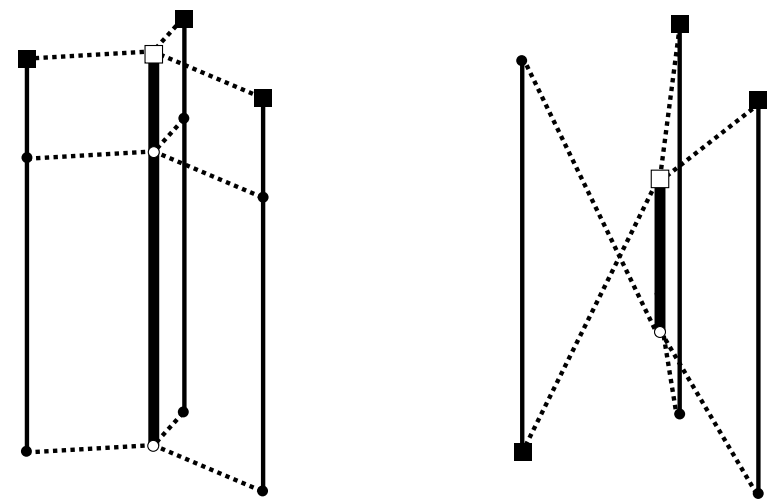

Figure 4: Principle generation of the center line. The average of all corresponding points forms the base point of the center line (left). In case of inverted lines, base point determination returns shifted coordinates towards the center point of the fiber bundle (right).

this algorithm is that it only works if all fibers have the same number of points and direction (Figure 4 right) which is not guaranteed by the tracking and clustering algorithms. To solve the problem of finding corresponding points, the algorithm performs resampling to guarantee the same number of points for all fibers. The number of sample points was defined as average number of points based on the original fibers. Subsequently, the sample points were linearly interpolated.

In order to find a consistent direction between starting and end points of the fibers for the whole cluster the algorithm starts with an arbitrary fiber and defines its direction for the whole cluster. Thereafter, the remaining fibers are adjusted after testing them against the direction of the cluster. The correct direction of a fiber is verified by comparing both endpoints and points in the middle of the fiber. This is necessary since in many cases cluster are of circular shape and endpoints fan out. We computed a similarity measure $m$ between two fibers which is the average of the euclidian distances of each corresponding pair of points. In the same way, the inverse similarity measure $i m$ was defined as $m$ with one fiber inverted. For $m$ larger then $i m$ the fibers have the same direction. Otherwise, they have to be aligned.

This procedure is sufficient, if there are no fibers that turn back. U-turns, which may be caused by tracking errors but can also be found in certain nerve tracts, are currently detected and excluded from the centerline computation. Up to now, this also applies to short fibers falling below a certain length threshold.

Normally, fibers of a bundle do not start and end at the same positions. The centerline thus leaves the center of the cluster which is not a serious problem since the centerline is only used as a tool for the definition of the intersection planes (see section 5.2) and is not directly involved in the visualization process.

\subsection{Boundary curves}

After the determination of the center line, a basis for the final mesh generation must be generated. This was achieved by computing $2 \mathrm{D}$ boundary curves, marking the desired boundary of the wrapped tract system. For this purpose, we defined planes orthogonal to the line. The spacing between two planes was chosen equidistant. The distance between neighboring planes has to be small enough to avoid distinctive discontinuities where the single segments join. After defining the base point $t_{b}$ for each plane on the center line the corresponding tangent vector is calculated and used as normal for
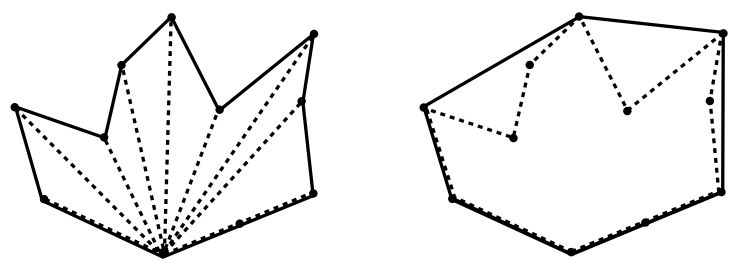

Figure 5: Schematic outline of the Graham's Scan algorithm for the generation of the convex hull.

of the fiber bundle for each plane. In a first step, the intersection point $t_{i}$ of each fiber with the plane is calculated. If the cluster is strongly curved, a fiber can intersect the plane more than once. In this case, we only used the intersection point closest to $t_{b}$.

A subset of points is then determined depending on the variance of the location of the points. Geometrically, the value selected for variance thresholding expresses the extension of the hull around the center line. From a surgical point of view this is a parameter of safety. Defining a higher variance leads to the incorporation of more fibers which extends the size of the fiber tract. It is also useful to provide the possibility to extend the boundary curve and expand the enclosed area. This parameter strongly depends on parameters of fiber tracking and bundling. Defensive tracking and bundling will decrease safety zones while an offensive choice of parameters reduces accuracy of the tract system. These limitations cannot be overcome with improved variance selection but influence it.

The resulting set of points serves as input for the Graham's Scan algorithm [16] we used to generate the convex hull. This algorithm first searches for an outer point in the set as base point $q$ by comparing coordinates. Afterwards, an edge connecting $q$ with an arbitrary second point from the set is chosen as reference. Consecutively, the angles between the reference edge and each connection to other points in the set are derived and sorted in ascending order. Points with the same corresponding angle are sorted ascending by their distance to $q$. By connecting all points in the obtained order the algorithm achieves a star-shaped polygon which avoids crossing edges. Finally, concave corners are skipped leading to the requested convex hull (Figure 5).

Instead of the convex hull, it is also possible to determine ellipses of smallest areas as boundary curves. As an advantage, they have a smoother surface. The major disadvantage a elliptical hull is the worse fitting surface around the fibers. Ellipses may feature large gaps between the border and the included fibers. Depending on the shape of the tract this disadvantage is more or less serious. If accuracy is of great interest, ellipses should be avoided.

\subsection{Mesh generation}

The task of generating a mesh out of several boundary curves is well known [14]. Here, we implemented a simplified approach fitting the requirements while benefit from the preconditions of our curves. For rendering the hull, it is advantageous to use triangle strips due to performance issues. Thus, a regular mesh has to be generated. As a drawback, the computed bounding curves usually do not have the same number and distribution of points as required for this type of mesh. Therefore, an algorithm is used which resamples the curve to equalize the number of points to a previously defined value (Figure 6). The algorithm places the new points by following the bounding curve creating points after each step with length $l$. Thereby, distance $l$ is computed by dividing the length of the curve by the number of desired points. It must be pointed out 


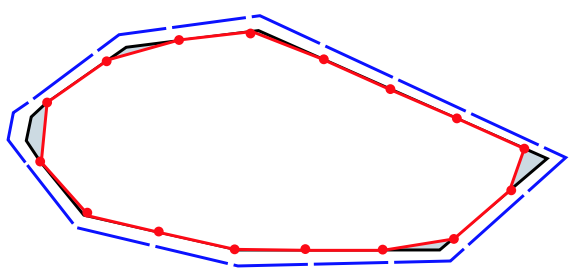

Figure 6: Resampling of the bounding curve. The blue line denotes the equidistant steps. The red line represents the resulting bounding curve. Gray areas are clipped.

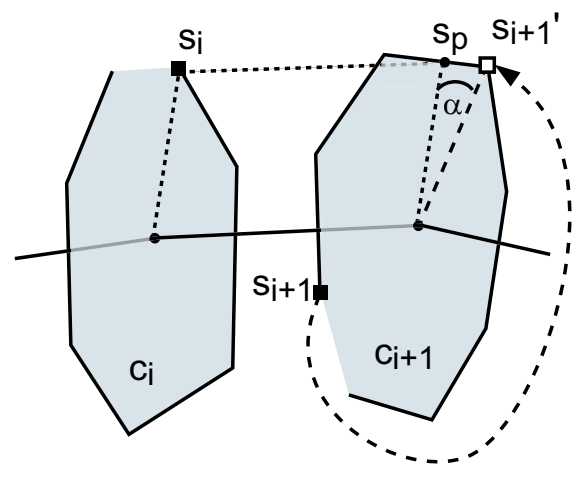

Figure 7: Determination of the new starting point $s_{i+1}{ }^{\prime}$ on curve $c_{i+1}$ by projection of $s_{i}$ to $s_{p}$ and succeeding search of the best fitting curve point on $c_{i+1}$.

that a certain loss of accuracy is associated with the use of this algorithm since corners of the original polygon are clipped. By choosing a sufficiently small $l$ the produced error is acceptable. The next step is to search corresponding points on two neighboring curves $c_{i}$ and $c_{i+1}$ (Figure 7). First, the algorithm determines a temporary point $s_{p}$ on curve $c_{i+1}$ which corresponds to the starting point $s_{i}$ on curve $c_{i}$. Afterwards, determination of the remaining point pairs is simple due to equivalent winding generated by the Graham's Scan algorithm and the nearly equidistant distribution of the points after resampling. For the search of $s_{i+1}{ }^{\prime}, s_{i}$ is projected to $s_{p}$ in the plane of curve $c_{i+1}$ along the vector from the barycenter of curve $c_{i}$ to the barycenter of curve $c_{i+1}$. The algorithm selects the point $s_{i+1}{ }^{\prime}$ on curve $c_{i+1}$ as the new starting point which encloses the smallest angle $\alpha$ with $s_{i+1}$ and the barycenter of $c_{i+1}$. The corresponding normals per vertex are computed by averaging the normals of the six neighboring triangles.

A special case occurs, if two consecutive planes are intersecting each other in the range of their corresponding boundary curves. In this situation, the intersection line is determined and a filling mesh is inserted on the side with correct plane order analogous to a standard segment.On the other side with changed plane order a medial plane is calculated which serves as a basis for a combined boundary curve connecting the two neighboring segments.

\section{Visualization}

All the rendering is based on the OpenGL API. Since surfaces were stored as triangle strips using vertex arrays convenient rendering could be applied. The application is embedded into a software en- vironment for the analysis and visualization of medical image data which uses Open Inventor for rendering management and $Q t$ for the GUI which enables user interaction.

Combining the wrapped fibers and direct volume rendering (DVR) of anatomical images is of great importance since simultaneous visualization reveals anatomical relations. Thereby, it is essential to use datasets matching each other. In almost every case the reference dataset, received during DTI acquisition, doesn't provide sufficient anatomical information due to low resolution and bad signal-to-noise ratio. On the other hand, other MRI sequences show very high anatomical detail. Therefore, it is desirable to combine the different data sources in a suitable manner. Assuming an aligned coordinate system for both, a DTI and an anatomical MRI dataset, they can be overlaid in a fused representation after registration in case of strong misalignment. In our case, we used consecutively scanned data which provided sufficient registration accuracy since patients were sedated. For the visualizations, hardware accel-

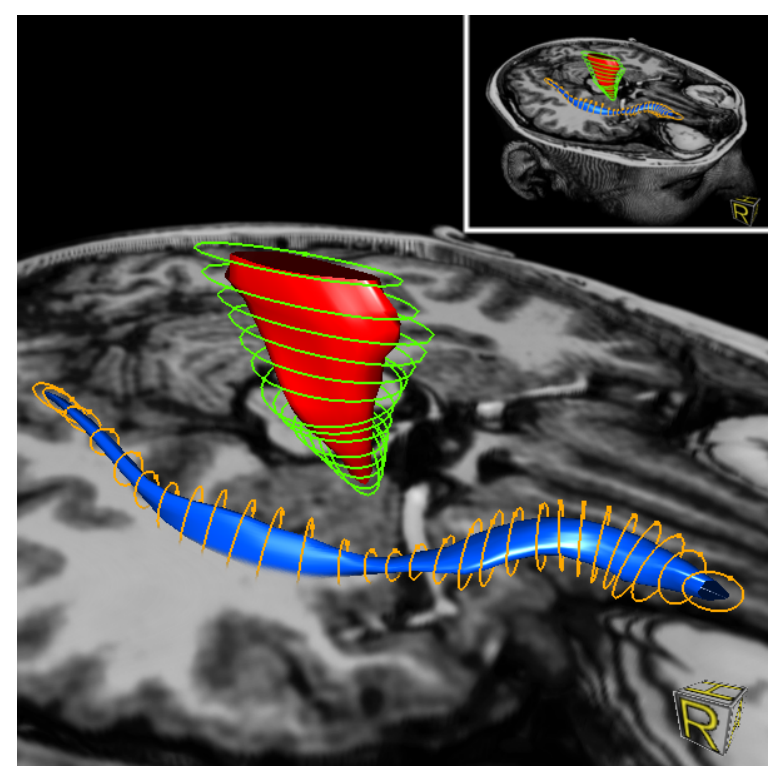

Figure 8: Combination of direct volume rendering of high resolution $M R I_{T 1}$ data with wrapped pyramidal and optic tract (red and blue respectively). The rings around the surfaces are more defensive approximations of the nerve tracts providing more security.

erated DVR [7, 28] was used exploiting advanced features such as flexible clipping [31] as well as 2D transfer functions (TF) [20].

Due to the opaque nature of the wrapping geometry, it is sufficient to execute the DVR after rendering the surface. Depth-testing prevents incorporation of occluded slices for volume rendering.

\subsection{Lighting}

Lighting plays an important role for the perception of the surface structure of the wrapped bundle. While surfaces based on ellipses are rather smooth, surfaces based on convex hulls may show small irregularities because of certain line features which cannot be recognized without shading. In contrast to that, lighting emphasizes such bumps and eases interpretation and evaluation of visualization in this application. Therfore, shading is essential for meaningful rendering. Gouraud shading already provides good results. Even better results can be achieved if per-pixel phong shading is applied (Figure 9). This can be accomplished with shaders that are supported by current graphics hardware. 


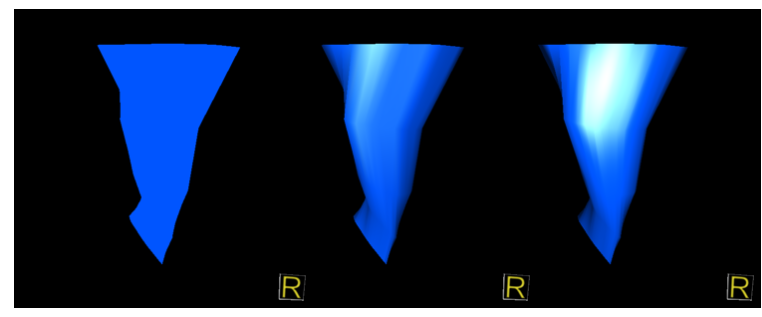

Figure 9: Wrapping of a pyramidal tract with the use of convex hulls rendered without lighting (left) with gouraud shading (middle) and per-pixel phong shading (right).

\subsection{Transparency}

Transparency is an excellent tool to compare the generated hulls with the underlying streamlines. The ability to observe both structures simultaneously enables the user to identify areas of uncertainty, expressed by less streamlines, while getting indications about possible dimension of a whole tract (see Figure 10). Thereby, semi-transparent rendering of a single hull is simple. After the opaque streamlines the wrapping surface is rendered by performing front-face culling in a first pass and back-face culling in a second pass. Due to the low amount of vertices (about 450 vertices per hull) the suggested 2-pass rendering approach is unproblematic. In fact, DVR is much more cost intensive than rendering of high resolution polygonal hulls due to fragment count and corresponding 3D-texture lookups. For the rendering of several hulls of the same

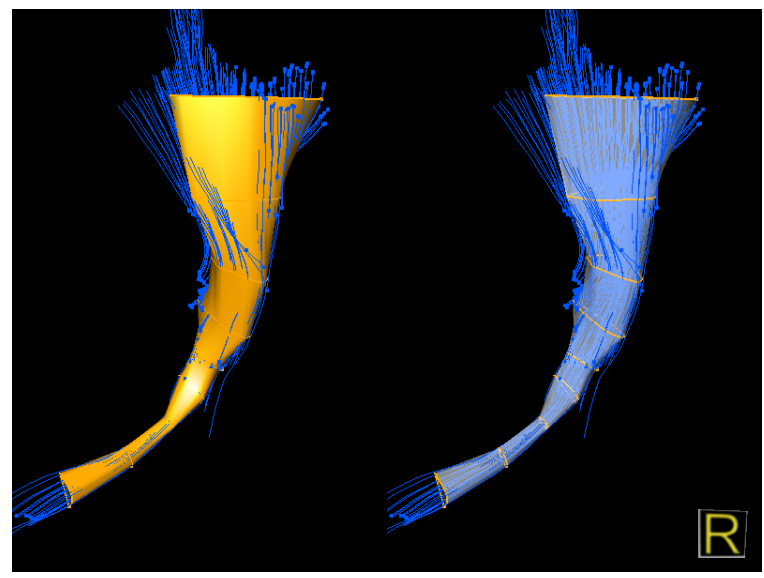

Figure 10: Rendering of opaque (left) and transparent (right) hull of the pyramidal tract in combination with streamlines of the corresponding fiber bundle. For the purpose of visual evaluation, semitransparent hulls are beneficial while opaque surfaces are more useful for an application in diagnosis.

bundle with different expansions this approach was extended. In the first pass the hulls were rendered with enabled front face culling in a descending order of expansion. Afterwards, ascending order and enabled back face culling were used. Thus, correct depth sorting is achieved (Figure 11).

\section{RESUlts AND Discussion}

The primary objective was the improvement of comprehensive nerve tract visualization which is accomplished by rendering lines

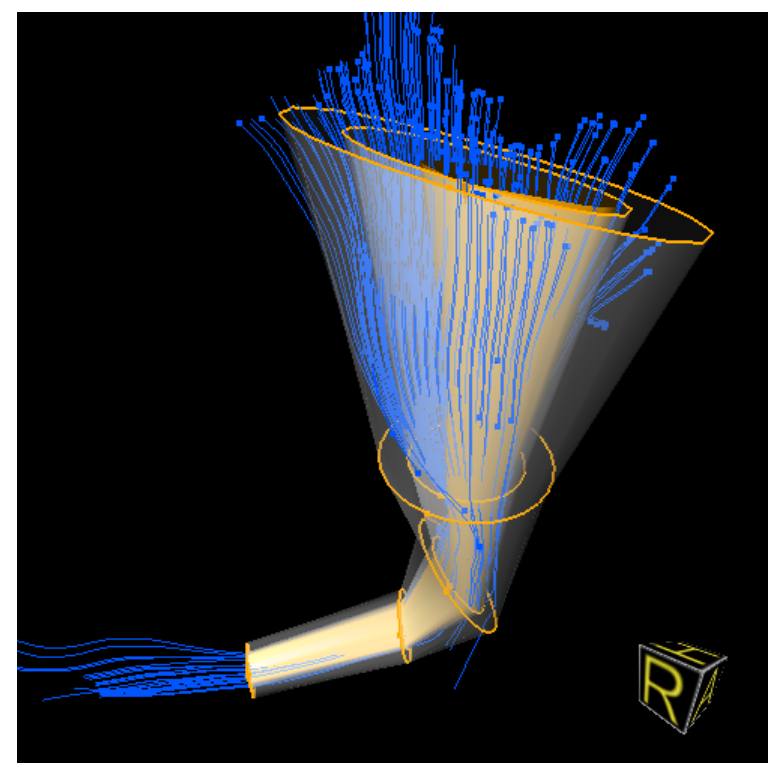

Figure 11: Combination of three hulls representing different amounts of enclosed fibers. The inner, opaquely rendered hull incorporates about $50 \%$ of the fibers. The medial hull covers approximately $70 \%$ of the fibers and the outer one wraps circa $90 \%$ of the them. Again, the same pyramidal tract bundle is used and ellipsoids are rendered for convenience. The larger diameter of the outmost hull at the top measures about $28.8 \mathrm{~mm}$.

in current applications. As a drawback, lines are limited in a way that they can only illustrate a direction. Additional information may be encoded in color or, in case of extensions like (hyper-) streamlines, in shape but still geometrical features of an underlying structure can only be estimated. On the other hand, the actual problem is the comprehensive display of the very geometric structure of nerve tracts, especially in neurosurgery.

The basis for the wrapping approach are bundles of fibers resulting from tractography and clustering. Fiber tracking was performed for the whole brain with a threshold of about 0.35 for FA. The average time for this preprocessing step of a $128 \times 128 \times 60$ DTI dataset was about 60 seconds on a P4 $3.0 \mathrm{GHz}$ with $2 \mathrm{~GB}$ RAM. Since tractography provides only an unsorted set of lines, bundling has to be performed in a second step. The general decision whether using manual or automatic bundling depends on the application field. For the analysis of a single tract system, ROI based bundling is preferable. Firstly, it is much faster compared to automatic bundling and secondly, expert knowledge is incorporated into the process leading to a much better selection of relevant fibers. In case of exploration of the tracking of a while brain, automatic clustering provides sufficient results. Without user interaction clustering generates bundles based only on geometric features of the fibers. Of course this also means that yet no medical expertise is employed. Additionally, clustering for a whole brain is very time and resource consuming. It took up to 10 minutes to generate a complete bundling of a brain consisting of about 4000 fibers. Especially, clustering based only on geometrical features needs further investigation.

The comprehensive display is achieved by wrapping surfaces around bundles. Figure 12 shows this by means of a case of the pyramidal tract situated very close to a tumor. It can be seen that the wrapping accomplishes the task of visualizing the appearance of the nerve tract in a suitable manner and supports better differentiation between abnormal and healthy tissue. Lines are not capable to provide an easy to interprete border.

Special attention has to be paid to the amount of fibers incor- 

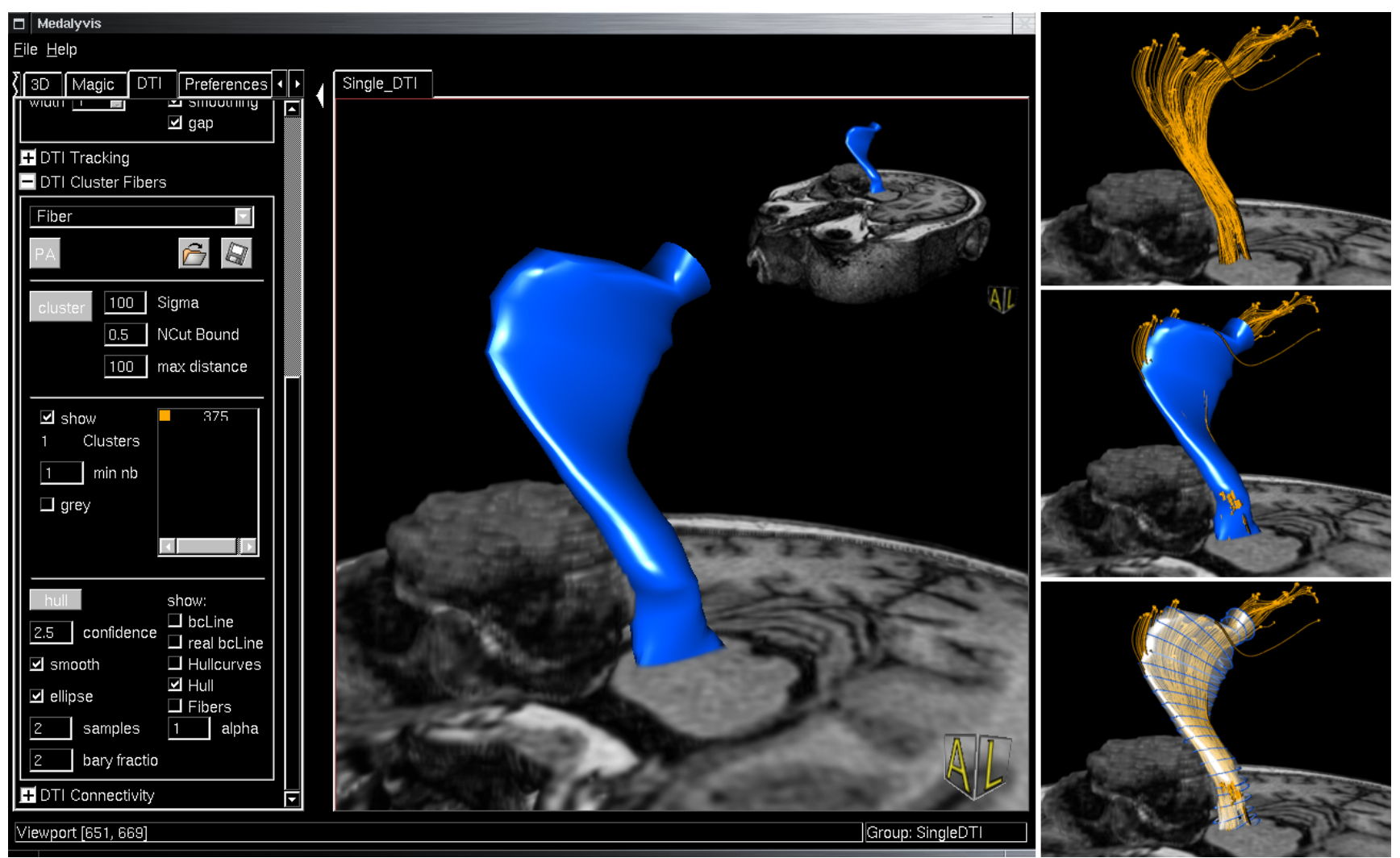

Figure 12: Screenshot of our application (left) showing a case of glioma disease with the tumor emphasized by segmentation. The pyramidal tract (blue) is situated close to the area designated for resection. Hull visualization enables clear identification of the transition between tissues which helps to protect injury of the tract. For line representation (upper right) this is more ambiguous. Combined rendering of the hull and the fibers (lower right) clarify the fitting of the surface and show the reliability of the hull.

porated into the wrapping. This parameter influences the extension of the received surface and is directly related to the underlying tractography. Offensive fiber tracking leads to wide spread bundles most likely extending the borders of the nerve tract. On the other hand, defensive tracking will eventually miss some areas of the tract which has to be compensated. This can be achieved by adjusting the corresponding threshold.

Handling of the end section of fiber bundles is a critical point of wrapping. Most of the bundles fan out at their end, leading to sparse density of intersection points for the convex hull computation. In this case, the expressiveness of wrapped bundles decreases and line rendering is preferable. Therefore, wrapping of fibers is stopped by a threshold of the variance of the fiber distribution. Since the blurry characteristic originates from the DTI data itself this behavior is acceptable. Further processing would lead to misleading visualization.

Transparency enables the comparison of different experiments. Since parameters for wrapping are not identical for all setups, it is still necessary to tune them. Thereby, visual evaluation of the results is enhanced by the use of transparent hulls. Simultaneous rendering of fibers of a bundle and the corresponding hull allows rating of the surface due to gaps and penetration between fibers and hull, now visible for the user. Furthermore, several hulls of the same bundle can be displayed providing the user with additional information. In clinical application, this may be used to offer several alternatives to choose for medical evaluation. This is directly related to DVR. Merging nerve tract visualization with DVR of high resolution MR data has great potential. Spatial relation, which is of special interest, between structures is emphasized and hence better to interpret due to the direct visual access. It must be pointed out that this approach requires clipping as a prerequisite since the visualization of MRI data is very difficult to optimize by the use of 1D transfer function. Even multidimensional transfer functions are currently insufficient to extract relevant structures and to define transparency in a way that a meaningful $3 \mathrm{D}$ volume rendering is obtained.

\section{Conclusion}

We have presented a new technique for comprehensive visualization of nerve tracts. The development was encouraged by the demands of neurosurgery where visualization of white matter tracts as geometric objects is of high value. Our solution comprises a twostep process extracting tract systems from DTI data and generating surfaces for a geometric representation. Extraction is achieved by bundling fibers produced by tractography either manually or automatically by analyzing geometric features of the fibers. Afterwards, a surface is generated which wraps the desired fibers. Finally, we applied lighting and transparency to the rendering and combined it with DVR for anatomical orientation purpose.

Overall, a more comprehensive visualization for DTI fiber tracts is presented which enriches medical applications such as intervention planning in neurosurgery. 


\section{Future WORK}

Automatic clustering of fibers is a valuable alternative to ROI tracking which still has potential for improvement such as the incorporation of further geometrical features and medical knowledge. In the future, the visualization will be improved by additionally using concave hulls which would further increase accuracy of the resulting representations of the nerve tracts. Furthermore, the obtained wrappings will be adapted for the incorporation into neuronavigation and the operating microscope in the context of an augmented reality approach.

\section{ACKNOWLEDgments}

This work was supported by the Deutsche Forschungsgemeinschaft in the context of SFB 603, Project C9 and the Graduate Research Center "3D Image Analysis and Synthesis".

\section{REFERENCES}

[1] David Akers, Anthony Sherbondy, Rachel Mackenzie, Robert Dougherty, and Brian Wandell. Exploration of the brain's white matter pathways with dynamic queries. In Proc. IEEE Visualization, pages 377-384, 2004.

[2] P. J. Basser, S. Pajevic, C. Pierpaoli, J. Duda, and A. Aldroubi. In vivo fiber tractography using DT-MRI data. Magn. Reson. Med., 44:625632, 2000.

[3] P.J. Basser, J. Mattiello, and D. LeBihan. MR diffusion tensor spectroscopy and imaging. Biophys. J., 66(1):259-267, 1994.

[4] Mats Bjornemo, Anders Brun, Ron Kikinis, and Carl-Fredrik Westin. Regularized stochastic white matter tractography using diffusion tensor MRI. In Proc. MICCAI, pages 435-442, 2002.

[5] Anders Brun, Hans Knutsson, Hae-Jeong Park, Martha E Shenton, and Carl-Frederik Westin. Clustering fiber tracts using normalized cuts. In Proc. MICCAI, pages 368-375, 2004.

[6] Steve Bryson and Creon Levit. The virtual windtunnel: An environment for the exploration of three-dimensional unsteady flows. In Proc. IEEE Visualization, pages 17-24, 1991.

[7] B. Cabral, N. Cam, and J. Foran. Accelerated volume rendering and tomographic reconstruction using texture mapping hardware. In Proc. Symposium on Volume Visualization (VVS), pages 91-98, 1994.

[8] Thierry Delmarcelle and Lambertus Hesselink. Visualizing secondorder tensor fields with hyperstreamlines. IEEE Comput. Graph. Appl., 13(4):25-33, 1993.

[9] Zhaohua Ding, John C. Gore, and Adam W. Anderson. Case study: reconstruction, visualization and quantification of neuronal fiber pathways. In Proc. IEEE Visualization, pages 453-456, 2001.

[10] Zhaohua Ding, John C. Gore, and Adam W. Anderson. Classification and quantification of neuronal fiber pathways using diffusion tensor MRI. Magn. Reson. Med., 49:716-721, 2003.

[11] Albert Einstein. Über die von der molekularkinetischen Theorie der Wärme geforderte Bewegung von in ruhenden Flüssigkeiten suspendierten Teilchen. Annalen der Physik, 17:549-560, 1905.

[12] Lisa Jonesa Elias R. Melhema, Ryuta Itoha and Peter B. Barkera. Diffusion tensor MR imaging of the brain: Effect of diffusion weighting on trace and anisotropy measurements. Am. J. Neuroradiol., 21:1813$1820,2000$.

[13] Frank Enders, Sabine Iserhardt-Bauer, Peter Hastreiter, Christopher Nimsky, and Thomas Ertl. Hardware-accelerated glyph based visualization of major white matter tracts for analysis of brain tumors. In Proc. SPIE Medical Imaging, 2005.

[14] H. Fuchs, Z. M. Kedem, and S. P. Uselton. Optimal surface reconstruction from planar contours. Commun. ACM, 20(10):693-702, 1977.

[15] Marcel Gavriliu, Joel Carranza, David E. Breen, and Alan H. Barr. Fast extraction of adaptive multiresolution meshes with guaranteed properties from volumetric data. In Proc. IEEE Visualization, pages 295-303, 2001.
[16] Ronald L. Graham. An efficient algorithm for determining the convex hull of a finite planar set. Inf. Process. Lett., 1(4):132-133, 1972.

[17] Gordon Kindlmann. Superquadric tensor glyphs. In Proc. Eurographics - IEEE TCVG Symposium on Visualization, pages 147-154, 2004.

[18] Gordon Kindlmann and David Weinstein. Hue-balls and lit-tensors for direct volume rendering of diffusion tensor fields. In Proc. IEEE Visualization, pages 183-189, 1999.

[19] Gordon Kindlmann, David Weinstein, and David Hart. Strategies for direct volume rendering of diffusion tensor fields. IEEE Trans. Vis. Comput. Graph., 6(2):124-138, 2000.

[20] Joe Kniss, Gordon Kindlmann, and Charles Hansen. Interactive volume rendering using multi-dimensional transfer functions and direct manipulation widgets. In Proc. IEEE Visualization, pages 255-262, 2001.

[21] M. Lazar, D.M. Weinstein, J.S. Tsuruda, K.M. Hasan, K. Arfanakis, M.E. Meyerand, B. Badie, H.A. Rowley, V. Haughton, A. Field, and A.L. Alexander. White matter tractography using diffusion tensor deflection. Hum. Brain Mapp., 18(4):306-321, 2003.

[22] N.F. Lori, E. Akbudak, J.S. Shimony, T.S. Cull, A.Z. Snyder, R.K. Guillory, and T.E. Conturo. Diffusion tensor fiber tracking of human brain connectivity: aquisition methods, reliability analysis and biological results. NMR Biomed., 15(7-8):494-515, 2002.

[23] S. Mori, B.J. Crain, V.P. Chacko, and P.C. van Zijl. Three dimensional tracking of axonal projections in the brain by magnetic resonance imaging. Ann. Neurol., 45(2):265-269, 1999.

[24] S. Mori and P. van Zijl. Fiber tracking: principles and strategies - a technical review. NMR Biomed., 15:468-480, 2002.

[25] C. Nimsky, O. Ganslandt, P. Hastreiter, R. Wang, T. Benner, A.G. Sorensen, and R. Fahlbusch. Preoperative and intraoperative diffusion tensor imaging-based fiber tracking in glioma surgery. Neurosurgery, 56(1):130-138, 2005.

[26] S. Pajevic, A. Aldroubi, and P.J. Basser. A continuous tensor field approximation of discrete DT-MRI data for extracting microstructural and architectural features of tissue. J. Magn. Reson., 154(1):85-100, 2002.

[27] C. Poupon, C. A. Clark, V. Frouin, J. Régis, I. Bloch, D. Le Bihan, and J.-F. Mangin. Regularization of diffusion-based direction maps for the tracking of brain white matter fascicles. Neuroimage, 12(2):184-195, 2000.

[28] Christoph Rezk-Salama, Klaus Engel, Michael Bauer, Günther Greiner, and Thomas Ertl. Interactive volume rendering on standard $\mathrm{PC}$ graphics hardware using multi-textures and multi-stage rasterization. In Proc. ACM SIGGRAPH/EUROGRAPHICS workshop on Graphics hardware, pages 109-118, 2000.

[29] Jianbo Shi and Jitendra Malik. Normalized cuts and image segmentation. IEEE Trans. Pattern Anal. Mach. Intell., 22(8):888-905, 2000.

[30] David M. Weinstein, Gordon L. Kindlmann, and Eric C. Lundberg. Tensorlines: Advection-diffusion based propagation through diffusion tensor fields. In Proc. IEEE Visualization, pages 249-254, 1999.

[31] Daniel Weiskopf, Klaus Engel, and Thomas Ertl. Interactive clipping techniques for texture-based volume visualization and volume shading. IEEE Trans. Vis. Comput. Graph., 9(3):298-312, 2003.

[32] C.-F. Westin, S. E. Maier, H. Mamata, A. Nabavi, F. A. Jolesz, and R. Kikinis. Processing and visualization of diffusion tensor MRI. Med. Image Anal., 6(2):93-108, 2002.

[33] Burkhard C. Wünsche and Richard Lobb. The visualization of diffusion tensor fields in the brain. In Proc. Int. Conf. on Mathematics and Engineering Techniques in Medicine and Biological Science, METMBS, pages 498-504, 2001.

[34] Song Zhang, Cagatay Demiralp, and David H. Laidlaw. Visualizing diffusion tensor $\mathrm{mr}$ images using streamtubes and streamsurfaces. IEEE Trans. Vis. Comput. Graph., 9(4):454-462, 2003. 